\title{
Ventriculo-peritoneal shunt trans-anal protrusion causing Escherichia coli ventriculitis in child: Case report and review of the literature
}

\author{
Yuhan Liu', Chunde Li ${ }^{1}$ and Yongji Tian ${ }^{1,2,3^{*}}$
}

\begin{abstract}
Background: Ventriculo-peritoneal shunting is an effective treatment for hydrocephalus, bowel perforation by peritoneal catheters is an extremely rare complications associated with this procedure.

Case presentation: We report a 9-year-old girl who had anal VP shunt protrusion two months following surgery and review the literature to understand the diagnosis and treatment for this rare complication. A PubMed search using the keywords 'bowel perforation' and 'Escherichia coli ventriculitis' was performed and citations were reviewed.
\end{abstract}

Conclusions: Neurosurgeons should be alert to early diagnosis and appropriate treatment of this condition to prevent infectious and neurological complications.

\section{Background}

Bowel perforation by peritoneal catheter (BPPC) is a kind of rare complication that could occasionally occur after ventriculo-peritoneal shunt (VPS) procedure and could result in potentially fatal ventriculitis or sepsis [1]. Patients suffering from BPPC do not always present with significant abdominal symptoms (less than $25 \%$ ) such as peritonitis. Awareness and early recognition of this complication are essential because of the high mortality rate in such patients (15\%) [2]. Until now, the literature across the world has been reported cases on 112 patients with BPPC, and more than half of the cases occurred within the age group of $0-10$ years. Here, we report on a 9-year-old girl who had an anal VP shunt protrusion 2 months following surgery with Escherichia coli ventriculitis and with no signs of peritonitis, bowel obstruction, or VPS malfunction, and we review the literatures

\footnotetext{
*Correspondence: tyjpumc@163.com

'Department of Neurosurgery, Beijing TianTanHospital, Capital Medical University, Beijing 100050, People's Republic of China

${ }^{2}$ China National Clinical Research Center for Neurological Diseases

(NCRC-ND); Center for Brain Tumor, Beijing Institute for Brain Disorders; Beijing Key Laboratory for Brain Tumor, Beijing 100050, People's Republic of China

Full list of author information is available at the end of the article
}

on this rare but serious complication. Our goal is to alert physicians and neurosurgeons to the early diagnosis and treatment of this complication to minimize or prevent infectious and neurological consequences.

\section{Case presentation}

A 9-year-old girl diagnosed with germinoma in the third ventricle and obstructive hydrocephalus underwent VP shunting at the end of December 2015 in our hospital. She was given chemotherapy and noticed the catheter protruding through the anus one day before presentation in our emergency department in January 2016.

She presented with fever, headaches and vomiting. Physical examination showed she was alert but weak, and fundoscopy did not reveal papilledema. She exhibited signs of ventriculitis of neck stiffness, but she had no abdominal pain, her abdomen was soft, and no catheter was observed in the anal sphincter area or palpated during a rectal exam.

The abdominal X-ray did not show any shunt fracture or free abdominal air but confirmed the penetration of the distal (peritoneal) catheter of the VP shunt into the bowel as well as its trajectory from the abdomen towards the perineal region (Fig. 1). Computed 

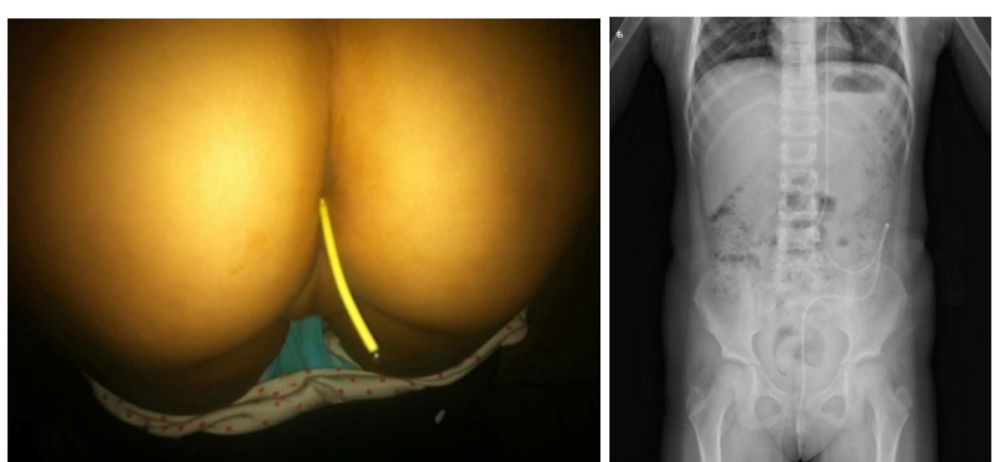

Fig. 1 Left: Picture of the distal peritoneal catheter of the ventriculoperitoneal shunt protruding through the patient's anal region. Clear CSF drops could be seen at the end of the catheter. Right: Plain abdominal x-ray confirming bowel perforation by the distal peritoneal catheter as well as its trajectory from the abdominal cavity toward the perineal region

tomography $(\mathrm{CT})$ of the abdomen revealed that the distal catheter penetrated the descending colon and followed the bowel towards the sigmoid colon (Fig. 2)

After the neurosurgery team discussion, the patient was operated to get the proximal (ventricular) catheter externalized. During the operation, an incision was created over the distal shunt on the abdominal wall. The peritoneal catheter was disconnected from the proximal portion and then removed from the distal portion without resistance. The proximal catheter was connected to a collecting bag. A post-operative abdominal ultrasound revealed no complications. Cerebrospinal fluid (CSF) analysis revealed a pattern suggestive of bacterial meningitis (leukocytes 5956/ul, glucose $1.7 \mathrm{mmol} / \mathrm{L}$, and protein $117 \mathrm{~g} / \mathrm{L})$. The cultures of both the initial CSF sample and the cranial tip of the VP shunt revealed Escherichia coli, and treatment with the broad-spectrum antibiotic meropenem was initiated. CSF tests were performed daily. The child have a rapid, uneventful recovery without fever and CSF cultures became negative in ten days. After the end of the antibiotic treatment and three negative cultures, a repeated MRI scan revealed the tumour shrinkage and no signs of hydrocephalus after the distal catheter was clipped for over $48 \mathrm{~h}$, and the whole VP shunt system was removed. The girl recovered uneventfully without additional antibiotic treatments and get back to her baseline condition for the last 4 months.

She was in good condition, and a repeated MRI revealed no signs of the tumour after chemotherapy at the last follow-up.

\section{Discussion}

Bowel perforation caused by a VPS tube is pretty rare with an estimated incidence from $0.1 \%$ to $1.0 \%$ in shunted patients $[3,4]$. However, this condition could cause devastating complications, such as meningitis and ventriculitis and carries a mortality rate of approximately $15 \%$ [2]. The first case of bowel perforation was reported in 1966 [5]. The shunt abdominal catheter may protrude from the large bowel [6], urinary bladder [7], urethra [8], or even the oral cavity [9]. The most common perforation site is the colon [10].

In a recently published review by Hai et al. [11], anal protrusions of distal shunt catheters were reported in 96 patients. Since that time, 15 cases have been reported [7, $8,12-23]$. In total, there are 112 cases including our case. Among these 112 cases, the ages of the 57 cases ranged from 0 to 10 years, and our patient belonged to this group. We think that the number of reported patients with this complication may have been higher if we had included articles published in non-indexed journals

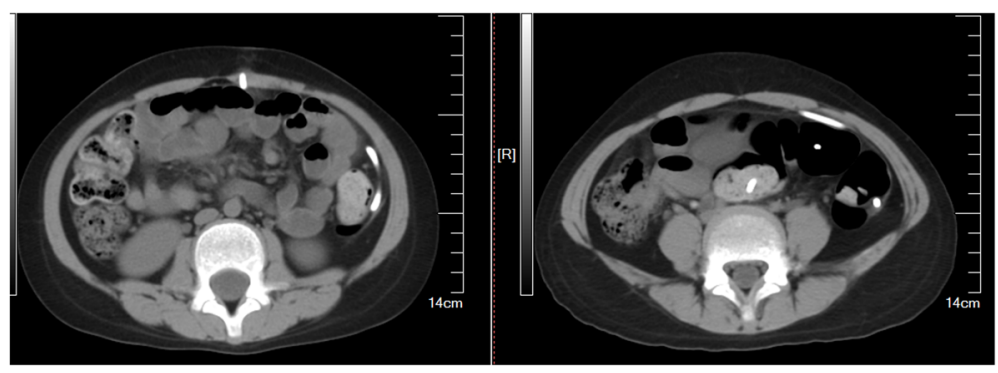

Fig. 2 Abdominal computed tomography scan demonstrating the migration peritoneal catheter in the bowel lumen 
[13]. The interval between the shunt operation and the occurrence of the anal shunt protrusion ranges from weeks to several months. In our case, this interval was nearly two months.

More than $50 \%$ of BPPC patients are asymptomatic, and the most common complaint is protrusion of the shunt catheter through the anus [24]. Among those with a catheter through the anus, many patients were diagnosed in an asymptomatic stage (42\%). The common symptoms are fever, abdominal pain, and vomiting $(40 \%)$, and the less common presentations are shunt malfunction, an abdominal abscess, and peritonitis.

The exact pathogenesis is difficult to discern. Akyuz et al. [25] hypothesized that the shunt catheter tip adheres to the viscera wall, thus forming the constant pressure of the abutting tip along with a local inflammatory reaction, finally leads to the erosion of the visceral wall and tip enter into the lumen. The peristaltic activity of the gut carries the catheter all the way down to the anus. Because it is usually a localized the inflammation, so, there are rarely any obvious signs of peritonitis.

Many authors have reported the formation of an encasing fibrosis around the catheter both at surgery and at autopsy, which is thought to have an anchoring effect on the tube that results in pressure on an area of the bowel. Pressure on the bowel area may finally cause the bowel to erode and result in perforation.

There are some other mechanisms been suggested in the literature, including weakening of the intestinal wall in a poor general condition patient and the stiff end of the shunt tube causing the perforation.

The use of a trocar during surgery may predispose patients to the occurrence of this complication. Other techniques for inserting peritoneal catheters include mini-laparotomy and laparoscopic-assisted insertion [26].

Techniques to investigate BPPC include abdominal Xray and CT [10, 27]. Plain $\mathrm{x}$-rays may confirm spontaneous bowel perforation because they can demonstrate the trajectory of the peritoneal VP-shunt catheter towards the perineal region. Abdomen CT scans are helpful to rule out the presence of an abdominal abscess. $\mathrm{X}$-rays, CT scans, and CSF cultures are positive in nearly $50 \%$ of cases.

In contrast, E. coli meningitis is also an indicator of intestinal perforation by a VP shunt tube [28].

Escherichia coli is the most common organism in BPPC cases. Based on the reported cases, the patients suffered from either ventriculitis or peritonitis, and some suffered both.

Once the diagnosis of BPPC has been established, the treatment should include total shunt removal, externalization of the ventricular drainage, and the broad-spectrum antibiotic therapy for at least 2 two weeks or until CSF cultures from the ventricles are negative.

The shunt catheter should be disconnected at the abdominal wall, and the distant end should be removed from the rectum assisted by colonoscopy or sigmoidoscopy [15]. The distal end of the V-P shunt should not be pulled back into the peritoneal cavity to prevent contamination of the tract. Because the bowel perforation caused by the shunt is usually very small, in most of the cases, it would seals by itself. Thus, laparotomy is rarely necessary and is indicated only for those patients who present with acute abdomen [11, 29, 30].

The treatment for early and late perforation may be quite different. For very acute (within a day) cases, it probably need an emergent operation to suture the perforation through laparotomy, while in chronic cases (from weeks to months after surgery), it maybe enough to remove the shunt as our case. Overall, most cases recovered very well and do not need an abdominal surgery to treat the bowel perforation.

Fortunately, our patient was, such as the majority of reported cases, asymptomatic and without any abdominal symptoms or signs. We hypothesize that the distal catheter perforated the descending colon, passed through the distal bowel, and exited out of the anus.

An external ventricular drainage should be kept for at least for 3 weeks, and patient should be given broadspectrum antibiotics to prevent/treat infections of the CSF. CSF infection due to the retrograde spread of the infection which may causing ventriculitis or meningitis should be ruled out after the extrusion of the shunt through the anus [19]. Once the CSF cultures are negative, a second shunting could be implanted if needed.

Antibiotics need to start to treat possible ventriculitis or meningitis causing by the retrograde spread of the infection prior to externalization $[3,31]$. The antibiotics that have been reported include linezolid, meropenem and metronidazole. The use of a sequential combination of intravenous antibiotics and intraventricular antimicrobial therapy (IVT) therapy has also been reported [32].

The possible factors responsible for BPPC are thin bowel walls in children, a sharp and stiff end of the V-P shunt [29], the use of a trocar by the operating surgeons [33], chronic irritation from the shunt, previous surgery, infection and silicone allergy [34].

Because of their weak bowel musculature, children are more susceptible to intestinal perforation. The use of modern soft and supple catheters composed of silicone that may incite a lesser foreign body reaction has been said to decrease the incidence of this complication.

Other techniques for inserting the peritoneal catheter, such as mini-laparotomy and laparoscopic-assisted insertion, may be helpful to prevent BPPC. However, large retrospective series have failed to demonstrate an advantage of one technique over the other in routine use [26]. 


\section{Conclusion}

We described the case of a 9-year-old girl with an anal protrusion of a VP shunt that presented 2 months following the shunt placement. Based on our case and a literature review, we stress the following issues: (a) the importance of early diagnosis and treatment to minimize or prevent infectious and neurological complications [7, 35], (b) the externalization of the proximal edge of the shunt without pulling the distal tip proximally by cutting the distal shunt tube over the abdominal wall and pulling the distal edge from the anal side to prevent the spread of infection spread, (c) the application of broad spectrum antibiotics until the CSF cultures are negative, and (d) the focus of attention on the abdominal symptoms and signs.

\section{Acknowledgements}

None.

\section{Funding}

Dr. Yongji Tian (Corresponding Author) was partially supported by the Beijing Municipal Administration of Hospitals' Youth Program QML20150501, National Key Technology Research and Development Program of the Ministry of Science and Technology of China (2014BAI04B01) and BIBD-PXM 2013_014226_07_000084.

\section{Availability of data and material}

Not applicable.

\section{Authors' contributions}

$\mathrm{CL}$ and $\mathrm{YT}$ conceived and designed the study. $\mathrm{YL}$ and $\mathrm{YT}$ wrote the paper. All authors read and approved the manuscript.

\section{Competing interests}

The authors declare that they have no competing interests.

\section{Consent for publication}

Informed consent was obtained for submission and publication of the case report from the parents of the respective patients.

\section{Ethics approval and consent to participate}

For this type of study formal consent is not required.

\section{Author details \\ 'Department of Neurosurgery, Beijing TianTanHospital, Capital Medical University, Beijing 100050, People's Republic of China. ${ }^{2}$ China National Clinical Research Center for Neurological Diseases (NCRC-ND); Center for Brain Tumor, Beijing Institute for Brain Disorders; Beijing Key Laboratory for Brain Tumor, Beijing 100050, People's Republic of China. ${ }^{3}$ Paediatric Neurosurgeon, Department of Neurosurgery, Beijing Tiantan Hospital, Beijing 100050, People's Republic of China.}

Received: 26 July 2016 Accepted: 8 December 2016

Published online: 06 February 2017

\section{References}

1. Panagea S, Cartmill TD, Panigrahi H. Intracerebral sepsis due to intestinal perforation by ventriculo-peritoneal shunts: two cases. J Infect. 1997;35:86-8.

2. Sathyanarayana S, Wylen EL, Baskaya MK, Nanda A. Spontaneous bowel perforation after ventriculoperitoneal shunt surgery: case report and a review of 45 cases. Surg Neurol. 2000;54:388-96.

3. Snow RB, Lavyne MH, Fraser RA. Colonic perforation by ventriculoperitoneal shunts. Surg Neurol. 1986:25:173-7.

4. Vinchon M, Baroncini M, Laurent T, Patrick D. Bowel perforation caused by peritoneal shunt catheters: diagnosis and treatment. Neurosurgery. 2006;58: ONS76-82. discussion ONS76-82.
5. Whittle IRJ. Extrusion of peritoneal catheter through neck incision: a rare complication of ventriculoperitoneal shunting. Aust N Z J Surg. 1983:53: $177-8$.

6. Shaw A, Lund JN, Semeraro D, Cartmill M, Reynolds JR, Tierney GM. Large bowel obstruction and perforation secondary to endometriosis complicated by a ventriculoperitoneal shunt. Colorectal Dis. 2008;10:520-1.

7. Mihajlovic M, Tasic G, Raicevic M, Mrdak M, Petrovic B, Radlovic V. Asymptomatic perforation of large bowel and urinary bladder as a complication of ventriculoperitoneal shunt: report of two cases. Srp Arh Celok Lek. 2012;140:211-5.

8. Yazar U, Kanat A, Akca N, Gazioglu G, Arda IS, Kazdal H. Urethral protrusion of the abdominal catheter of ventriculoperitoneal shunt: Case report of extremely rare complication. J Pediatr Neurosci. 2012;7:111-3.

9. Low SW, Sein L, Yeo TT, Chou N. Migration of the abdominal catheter of a ventriculoperitoneal shunt into the mouth: a rare presentation. Malays $J$ Med Sci. 2010;17:64-7

10. Peirce KR, LJ. Perforation of the intestine by a Raimondi peritoneal catheter. Case report. J Neurosurg. 1975;43:112-3.

11. Hai A, Rab AZ, Ghani I, Huda MF, Quadir AQ. Perforation into gut by ventriculoperitoneal shunts: A report of two cases and review of the literature. J Indian Assoc Pediatr Surg. 2011:16:31-3.

12. Oliveira SB, Monteiro IM. Endoscopic management of transanal protrusion of subdural peritoneal shunt in a child. J Pediatr Gastroenterol Nutr. 2011:53:465-7.

13. Borkar SA, Mahapatra AK. Ventriculoperitoneal shunt catheter protrusion through the anus. Childs Nerv Syst. 2012;28:341-2. author reply 343-344.

14. Glatstein M. Ventriculoperitoneal shunt catheter protrusion through the anus: case report of an uncommon complication and literature review. Childs Nerv Syst. 2012;28:343

15. Ozturk H, Is M, Ozturk H, Kucuk A, Dosoglu M. Transanal protrusion of a ventriculoperitoneal shunt catheter. J Coll Physicians Surg Pak. 2012;22:733-4.

16. Aras $M$, Altas M, Serarslan $Y$, Akcora B, Yilmaz A. Protrusion of a peritoneal catheter via abdominal wall and operated myelomeningocele area: a rare complication of ventriculoperitoneal shunt. Childs Nerv Syst. 2013;29:1199-202.

17. Mattei TA, Salma A, Lin JJ. Spontaneous bowel perforation from distal catheter leading to meningitis: a rare but frequently overlooked complication of ventriculoperitoneal shunts. Pediatr Neurol. 2013:48:477-8.

18. Rinker EK, Osborn DA, Williams TR, Spizarny DL. Asymptomatic bowel perforation by abandoned ventriculoperitoneal shunt. J Radiol Case Rep. 2013;7:1-8.

19. Sharifian A, Abdollahi A, Maddah G, Anaraki F, Alvandipour M, Abbasi Sahebi M, Bigdeli N, Hasanzadeh AM. Spontaneous transanal protrusion of ventriculoperitoneal catheter: a case report. Acta Med Iran. 2013;51:135-8.

20. Yilmaz MB, Egemen $E$, Tonge $M$, Kaymaz M. Transoral protrusion of a peritoneal catheter due to gastric perforation 10 years after a ventriculoperitoneal shunting: case report and review of the literature. Turk Neurosurg. 2013;23:285-8.

21. Plummer NR, Tokala A, Date RS. Transanal protrusion of ventriculoperitoneal shunt reflecting asymptomatic perforation of the large bowel. BMJ Case Rep. 2014:2014:bcr2014204842.

22. Voronovich ZA, Albright AL. Enterocutaneous fistula in the setting of ventriculoperitoneal shunt extrusion through the skin and perforation through the small bowel. J Neurosurg Pediatr. 2014;14:340-3.

23. Bansal H, Gupta G, Gupta M, Kaushal R. Unusual Ventriculoperitoneal (VP) Shunt Tube Extrusion through Anus in a Child with Dandy Walker Malformation: A Rare Case Report. J Clin Diagn Res. 2015;9:PD25-26.

24. Chiang LL, Kuo MF, Fan PC, Hsu WM. Transanal repair of colonic perforation due to ventriculoperitoneal shunt-case report and review of the literature. J Formos Med Assoc. 2010;109:472-5.

25. Akyüz MUT, Göksu E. A thoracic complication of ventriculoperitoneal shunt: symptomatic hydrothorax from intrathoracic migration of a ventriculoperitoneal shunt catheter. Br J Neurosurg. 2004;18:171-3.

26. Roth J, Sagie B, Szold A, Elran H. Laparoscopic versus non-laparoscopicassisted ventriculoperitoneal shunt placement in adults. A retrospective analysis. Surg Neurol. 2007:68:177-84.

27. Chung JJ, Yu JS, Kim JH, Nam SJ, Kim MJ. Intraabdominal complications secondary to ventriculoperitoneal shunts: $\mathrm{CT}$ findings and review of the literature. AJR Am J Roentgenol. 2009;193:1311-7.

28. Ibrahim AW. E. coli meningitis as an indicator of intestinal perforation by $\mathrm{V}$ P shunt tube. Neurosurg Rev. 1998;21:194-7.

29. Park CK, Wang KC, Seo JK, Cho BK. Transoral protrusion of a peritoneal catheter: a case report and literature review. Childs Nerv Syst. 2000;16:184-9. 
30. Vuyyuru S, Ravuri SR, Tandra VR, Panigrahi MK. Anal extrusion of a ventriculo peritoneal shunt tube: Endoscopic removal. J Pediatr Neurosci. 2009;4:124-6.

31. Zhou F, Chen G, Zhang J. Bowel perforation secondary to

ventriculoperitoneal shunt: case report and clinical analysis. J Int Med Res. 2007;35:926-9

32. Wang JH, Lin PC, Chou CH, Ho CM, Lin KH, Tsai CT, Wang JH, Chi CY, Ho MW. Intraventricular antimicrobial therapy in postneurosurgical Gramnegative bacillary meningitis or ventriculitis: a hospital-based retrospective study. J Microbiol Immunol Infect. 2014;47:204-10.

33. Jamjoom ABRJ, Kirkpatrick JN. Passage of tube per rectum: an unusual complication of a ventriculoperitoneal shunt. Br J Clin Pract. 1990;44:525-6.

34. Brownlee JDBJ, Schaefer IK. Colonic perforation by ventriculoperitoneal shunt tubing: a case of suspected silicone allergy. Surg Neurol. 1998:49:21-4.

35. Glatstein M, Constantini S, Scolnik D, Shimoni N, Roth J. Ventriculoperitoneal shunt catheter protrusion through the anus: case report of an uncommon complication and literature review. Childs Nerv Syst. 2011;27:2011-4.

Submit your next manuscript to BioMed Central and we will help you at every step:

- We accept pre-submission inquiries

- Our selector tool helps you to find the most relevant journal

- We provide round the clock customer support

- Convenient online submission

- Thorough peer review

- Inclusion in PubMed and all major indexing services

- Maximum visibility for your research

Submit your manuscript at www.biomedcentral.com/submit
Biomed Central 